clinic and 507 (99.2\%) of them had HIV test. From 42 patients having TOP, $6(14.2 \%)$ women had HIV test.

Discussion Not all patients might have been offered the HIV test in sexual health clinic. TOP service does not offer the HIV test routinely. Recommendations Sexual health clinic should offer HIV test to all patients accessing their service and document in patients' medical records that the HIV test was offered. When patients decline the HIV test, reasons for declining have to be documented. TOP service should update their local TOP guideline instructing their nurses, midwives and doctors to offer HIV test to all patients accessing their services. To present findings of the audit to medical staff at local, regional and national levels. To conduct re-audit in 3-6 months once changes have been implemented.

\section{P118 RAPE IN YOUNG ADOLESCENTS: RISK FACTORS FOR ATTENDING A SEXUAL ASSAULT REFERRAL CENTRE AND ENSURING ADEOUATE SEXUAL HEALTH FOLLOW-UP}

doi:10.1136/sextrans-2012-050601c.118

B Wilson-Brown, ${ }^{*}$ G Steele, F Fargie. The Archway, Sandyford

Background We are an urban sexual assault referral centre (SARC) also covering a large rural area. We see men and women over the age of 13 who have been sexually assaulted in the last 7 days. In 2011 we saw 53 attendences from adolescents between 13 and 15 years (13\% of all SARC attendences).

Aims (1) To review the profile of young victims of rape and sexual assault. (2) Retrospectively review the sexual and emotional health follow-up received by this client group post sexual assault. Method 2 data sets were reviewed. The SARC database were used to identify all clients attending in 2011 aged between 13 and 15 and to collect information including demographics/details of the assault/particular vulnerabilities including alcohol use and whether looked after and accommodated (LAAC) or known to social work. The National Sexual Health electronic patient record (NASH) was then used to review whether these clients attended for any sexual/emotional health follow from their local sexual health services to which they are routinely referred post assault. (A few clients requested referral to their GP.)

Results $32 / 53$ (60\%)of clients had been drinking alcohol prior to the assault. $14 / 53$ (26\%) of clients were LAAC and a further 12/53 (23\%) were known to social work. 5/53 clients had previously attended the SARC and were attending after a second sexual assault and all five clients were LAAC. 36/53 (67\%) had specialist sexual health input post assault with five clients due to attend in the next few weeks. Four clients were referred to their GP. Eight clients failed to attend any follow-up and social work were informed.

Conclusion A significant proportion of young adolescents attending our SARC have one or more markers for vulnerability. Health services need to be aware of the increased risk of sexual violence for these clients. Successful follow-up is possible with close liaison between health and social care workers.

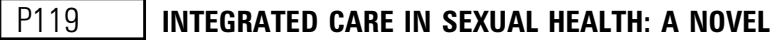 TRAINING PROGRAMME TO ENGENDER CULTURE CHANGE FOR TRAINEES IN PRIMARY AND SECONDARY CARE SPECIALTIES}

doi:10.1136/sextrans-2012-050601c.119

${ }^{1} \mathrm{C}$ Bradbeer, ${ }^{*}{ }^{2} \mathrm{~A}$ Mears, ${ }^{1} \mathrm{R}$ Kulasegaram. ${ }^{1}$ London Deanery; ${ }^{2}$ Imperial College Medical School

Background Changes in the NHS dictate that staff in general practice (GP), public health (PH), SRH and GUM work closely together.
This integration cannot be achieved by processes and structures alone. It requires a substantial change in attitude and understanding between the protagonists.

Aim To develop a training programme to increase trainees' understanding of the philosophy and ways of working of other disciplines involved in delivering sexual health care, so as to facilitate collaboration and integrated working.

Methods The day-long programme, first held in February 2011, was delivered to 21 specialist trainees reaching the end of their training in GUM, SRH, PH and GP. The London Deanery funded the innovation and invited participants from among its trainees. Each day was structured to cover: understanding population health; an update on the future of the NHS; workforce planning; involving patients and public; developing services, financial considerations and examining patient pathways. There was humorous stereotyping of specialties followed by group work to dispel misconceptions and understand each other's viewpoint. Speakers represented all invited disciplines and included a senior manager, a third sector leader and a member of the government's Listening Forum. A detailed evaluation of the day included an interval questionnaire after 4-8 months.

Results Two training days have now taken place; a third is oversubscribed for February 2012. Trainees said it was excellent, they found it inspiring and thought provoking. They requested more workshops which have been incorporated. Interval evaluations showed trainees had undertaken joint working and used their learning to prepare for consultant interviews. The model has now been used by London Deanery in related generic training programmes for other professionals and other disciplines.

Conclusion This model programme delivers effective training in leadership and culture change.

\section{P120 CORRELATION OF PATIENT COMPLETED TRIAGE TOOLS WITH PATIENT SYMPTOMS IN AN INTEGRATED SEXUAL HEALTH SERVICE}

doi:10.1136/sextrans-2012-050601c.120

S Bhaduri, ${ }^{*}$ C Gosling. Worcestershire Health and Care Trust, Worcestershire, UK

Background Triage tools (TT) were introduced in an integrated sexual health service to enable patients to be streamed to the most appropriate healthcare professional. The tool used is a patient completed questionnaire to ascertain reasons for attendance and any presenting symptoms.

Aim To determine whether the TT is a good indicator of patient symptoms and predictor of the skill mix required for the service.

Method A retrospective analysis of case notes reviewed gender, reported symptoms stated on TT and whether these symptoms correlated with the history taken from the patient.

Results 360 notes were analysed (178 females, 182 males), 125/178 $(70 \%)$ of females were symptomatic according to TT. In $17 / 178(9 \%)$ the correlation between TT and history taking was deficient. In 8/17 of these cases, issues related to contraception or referral for termination of pregnancy. 53/178 (30\%) were asymptomatic according to TT. In 13/53 cases, symptoms were later disclosed on history taking (dyspareunia, vulval discomfort, disclosure of assault or contraception issues). In males, 82/182 (45\%) were symptomatic and 7/ $182(4 \%)$ of cases demonstrated a correlation deficit (eg, disclosure of assault). 100/182 (54\%) of cases were asymptomatic on TT of which $24 / 100$ were not on history taking.

Conclusion In $61 / 360(17 \%)$ of patients the correlation of symptoms was incomplete on history taking compared to the TT. This indicates that although the tool remains a useful guide to patient presentation, clinics require a broad skill mix, including those with experience in dealing all aspects of integrated sexual health. 